\section{USO DEL TEAM BASED LEARNING PARA EL APRENDIZAJE DE BIOLOGÍA EN ESTUDIANTES DE MEDICINA}

\section{TEAM BASED LEARNING USE FOR BIOLOGY LEARNING IN MEDICAL STUDENTS}

\author{
Denisse Champin 1,a,b, Luis Huamán-Mesías ${ }^{1, c}$, \\ Arquímedes Gavino-Gutierrez ${ }^{1, d}$
}

Sr. Editor. En educación médica resulta fundamental el aprendizaje de ciencias básicas, puesto que constituyen las bases sobre las cuales se construirá el conocimiento clínico. Durante la formación médica, las materias básicas, a menudo, son percibidas por el estudiante como complejas y sin conexión con la carrera, habitualmente el estudiante no comprende la importancia del aprendizaje de temas debido a que los aprende fuera de contexto ${ }^{(1)}$.

El aprendizaje basado en equipos (TBL) es una metodología activa que está basada en el trabajo colaborativo, donde el estudiante desempeña un activo rol en su propio aprendizaje, guiado por un facilitador que cumple una función totalmente distinta de la del docente tradicional ${ }^{(2)}$. A diferencia de lo que ocurre en la educación tradicional, en la cual los escenarios de aprendizaje están limitados a las clases teóricas y las prácticas de laboratorio; en TBL, la construcción de conocimiento se realiza dentro del contexto, es decir, que el estudiante comprende la utilidad de aprender un tema porque lo aplica directamente a la solución de un caso planteado ${ }^{(3)}$. Esta metodología permite desarrollar competencias que son importantes para el futuro médico como la gestión de la información, el trabajo en equipo y el pensamiento crítico ${ }^{(2)}$.

En la presente comunicación se reportan los resultados obtenidos en el curso de Biología, cuando se agrega un espacio de TBL (modalidad híbrida), en la Escuela de Medicina de la Universidad Peruana de Ciencias Aplicadas (UPC). Se realizó un estudio transversal retrospectivo, aprobado por el Decanato de la Facultad

\footnotetext{
1 Escuela de Medicina, Universidad Peruana de Ciencias Aplicadas. Lima, Perú.

a Médico internista, ${ }^{\mathrm{b}}$ master en educación superior, ${ }^{\mathrm{c}}$ biólogo, ${ }^{\mathrm{d}}$ biólogo Recibido: 25-07-14 Aprobado: 06-08-14
}

Citar como: Champin D, Huamán-Mesías L, Gavino-Gutierrez A. Uso de team based learning para el aprendizaje de biología en estudiantes de medicina. Rev Peru Med Exp Salud Publica. 2014;31(3):601-2. de Ciencias de la Salud de la UPC. Posterior a un cambio curricular que modificó que el curso de Biología debía cursarse en paralelo con TBL, se crearon dos grupos en el ciclo 2010-2. Un grupo había llevado previamente TBL y, por ello, en dicho ciclo cursó Biología exclusivamente, y el otro grupo (modalidad híbrida) cursó Biología asociado a TBL. El curso de Biología tuvo el mismo contenido para ambos grupos.

El curso de TBL se desarrolla en tres sesiones y dura cuatro horas semanales, el número de alumnos por aula es treinta ${ }^{(4)}$. La estructura de TBL considera:

(a) Primera sesión: lectura o visualización de un video sobre un tema específico, se concluye con la entrega de una evidencia de haber participado activamente (resumen, mapa mental).

(b) Segunda sesión: evaluación individual sobre el tema de la primera sesión, posteriormente, se desarrolla en forma grupal la misma evaluación, a través de una discusión interna que ofrece aportes de distintos estudiantes. La sesión cierra con una discusión de los estudiantes con el facilitador acerca de la evaluación.

(c) Tercera sesión: el facilitador trae situaciones problemáticas (casos) que precisan que el alumno aplique el aprendizaje previo, se trabaja en grupos, estos preparan un producto (mapa mental) en el cual explican su propuesta de solución. Cada grupo presenta su propuesta de solución frente a la clase, a fin de recibir críticas. Para la solución de casos, se utiliza material de clases de Biología, textos y material de internet.

De los registros de la UPC se tomó, en forma anónima, las siguientes variables: sexo, nota final (escala vigesimal) del curso de Biología, carga curricular durante el ciclo 2010-2, y el número de veces que cursó Química y Biología. Para comparar las variables numéricas entre grupos se usó la t de Student y la suma de rangos de Wilcoxon según distribución normal. Para variables numéricas se utilizó el test exacto de Fisher. Se incorporaron 90 alumnos al estudio, 55 de ellos fueron mujeres $(61,1 \%)$. Cursaron la modalidad híbrida 62 alumnos (68,9\%); el 3,3\% llevó el curso por segunda vez. El promedio de notas de los alumnos del grupo sin TBL fue $11,8 \pm 1,4$, y el de la modalidad híbrida fue de $14,3 \pm 1,8$ ( $p<0,001)$, hubo diferencias en el número de cursos llevados durante el ciclo según modalidad de estudio $(p<0,001)$, no se encontró diferencias para las otras variables evaluadas (Tabla 1).

Considerando que los estudiantes de los dos grupos cursan Biología con la misma dinámica, los mismos docentes y la misma evaluación, el factor diferencial que nos podía explicar una marcada diferencia en 
Tabla 1. Diferencias entre las calificaciones de Biología y características entre los estudiantes de medicina que llevaron o no Team Based Learning (TBL)

\begin{tabular}{|c|c|c|c|}
\hline & Biología (\%) & Biología + TBL (\%) & $p$ \\
\hline Promedio final* & $11,8(1,4)$ & $14,3(1,8)$ & $<0,001 \ddagger$ \\
\hline Número de cursos** & $6(1,5)$ & $7(0)$ & $<0,001 \S$ \\
\hline \multicolumn{4}{|c|}{ Número de veces que lleva Biología } \\
\hline Primera & $26(92,9)$ & $61(98,4)$ & $0,230 \|$ \\
\hline Segunda/Tercera & $2(7,1)$ & $1(1,6)$ & \\
\hline \multicolumn{4}{|c|}{ Número de veces que lleva Química } \\
\hline Primera & $26(92,9)$ & $61(98,4)$ & $0,230 \|$ \\
\hline Segunda & $2(7,1)$ & $1(1,6)$ & \\
\hline
\end{tabular}

*Media (Desviación estándar), **Media(Rango intercuartil), ‡: T de student, §: Suma de rangos de Wilcoxon, II: Test exacto de Fisher.

los resultados de las notas, era el curso TBL, DONDE el estudiante desarrolla dinámicas de aprendizaje en equipos guiados por facilitadores, estos, a diferencia de lo que ocurre en la enseñanza tradicional, trabajan apoyando al alumno en su habilidad de análisis y razonamiento, aclarando malos entendidos, y de manera breve y concisa; se considera importante el aporte al aprendizaje proveniente de los compañeros de clase, los temas se revisan, se comparte información obtenida por cada uno de los miembros de la clase (sesión 2) y se proponen posibles soluciones al tema que se trata, las que son presentadas y discutidas de manera intergrupal (sesión 3); así, el estudiante logra un aprendizaje mayor del que podría alcanzar solo en consonancia con la teoría de desarrollo próximo que propone que la distancia entre el nivel real de desarrollo, determinado por la capacidad de resolver independientemente un problema, y el nivel de desarrollo potencial, determinado a través de la resolución de un problema bajo la guía de un adulto o en colaboración con otro compañero más capaz. Existen referencias de uso de TBL para el aprendizaje de ciencias básicas en medicina, para cursos como Física y Química, con resultados similares al nuestro ${ }^{(5)}$.

El diseño ideal para responder nuestra pregunta de investigación hubiera sido un estudio experimental, sin embargo, no es posible realizarlo dado que no se puede aleatorizar a los alumnos para que dejen de llevar un curso que es parte de la malla curricular. A pesar de ello, nuestro estudio permitió evidenciar que los alumnos que llevaron TBL tuvieron un mejor desempeño en el curso de Biología. El desarrollo de actividades grupales que incentiven la discusión y profundicen en temas desarrollados en materias regulares, son un coadyuvante para el aprendizaje.

Conflictos de interés: DC es directora de la Escuela de Medicina, LHM es coordinador del curso de Biología.

Financiamiento: autofinanciado

\section{REFERENCIAS BIBLIOGRÁFICAS}

1. Kaufman DM, Mann KV. Basic sciences in problem-based learning and conventional curricula: students' attitudes. Med Educ. 1997;31(3):177-80.

2. Michaelsen LK, Watson W, Cragin JP. Team learning: a potential solution to the problems of large classes. Exchange: The Organizational Behavior Teaching Journal. 1982;7(1):1822.

3. Wood DF. Problem based learning. BMJ. 2008 May 3;336(7651):971. doi: 10.1136/bmj.39546.716053.80.

4. Bermudez-García A, Mory-Arciniega C. Team BasedLearning (TBL) as a primary strategy for the development of generic competences in medical students: the Peruvian case. Med Teach. 2011;33(10):862.

5. Walters DE. Team-based learning applied to a medicinal chemistry course. Med Princ Pract. 2013;22(1):2-3. doi: 10.1159/000342819. Epub 2012 Sep 20.

Correspondencia: Denisse Champin Michelena

Dirección: Malecón Cisneros 1244-802. Miraflores. Lima, Perú

Teléfono: +5114456237

Correo electrónico:denisse.champin@upc.edu.pe 